\title{
Ear diseases among secondary school students in Xi'an, China: The role of portable audio device use, insomnia and academic stress
}

\author{
Yang Chen ${ }^{\dagger}, \mathrm{Xu} \mathrm{Li}^{\dagger}$, Zhan Xu, Zonghua Li, Pengzhi Zhang, Ya He, Fangyuan Wang and Jianhua Qiu*
}

\begin{abstract}
Background: Hearing impairment negatively impacts students' development of academic, language and social skills. Even minimal unilateral hearing loss can hinder educational performance. We investigated the prevalence of ear diseases among secondary school students in the city of Xi'an, China in order to provide a foundation for evidence-based hearing healthcare.

Methods: A stratified random sampling survey was conducted in 29 secondary schools. Demographics and medical histories were collected, and otologic examinations were performed. Questionnaires were administered to assess insomnia, academic stress and use of portable audio devices. Logistic regression analysis was used to identify factors associated with hearing impairment, and the association of sensorineural hearing loss with insomnia, academic stress and the use of portable audio devices was analyzed with the chi-square test.

Results: The percentage of students with some form of ear disease was 3.32\%. External ear disease, middle ear disease and sensorineural hearing loss occurred in 1.21\%, $0.64 \%$ and $1.47 \%$ of the students, respectively. Boys had a relatively higher prevalence of ear disease than girls. According to our survey, the prevalence of sensorineural hearing loss increased significantly among the students with insomnia and extended use of portable audio devices, but not among those with elevated levels of academic stress. Hearing aids and surgical treatment were needed in $1.47 \%$ and $0.89 \%$ of the students, respectively.
\end{abstract}

Conclusions: There is a high prevalence of ear disease among secondary school students, and this should be given more attention. Insomnia and the excessive use of portable audio devices may be related to adolescent sensorineural hearing loss. It is important to establish and comply with an evidence-based preventive strategy.

\section{Background}

Ear diseases may cause life-long or, sometimes, lifethreatening problems. Congenital or acquired hearing impairment (HI) hinders students' development of academic, language and social skills. Even minimal unilateral sensorineural hearing loss (SHL) can have a negative impact [1-4], and bilateral SHL can result in psychological problems. The World Health Organization (WHO) estimated that 278 million people in the world, two-thirds of whom were in developing countries, suffered from moderate to profound $\mathrm{HI}$ in 2005. It was also estimated that at least 68 million people have had

\footnotetext{
* Correspondence: qiujh@fmmu.edu.cn

† Contributed equally

Department of Otolaryngology-Head and Neck Surgery, Xijing Hospital,

Fourth Military Medical University, Xi'an-710032, China
}

HI since childhood [5]. These problems produce surprisingly large economic burdens on society as a whole. Therefore, early otologic diagnosis and intervention are important [6].

Due to the impact of HI on mental and physical health, the WHO formulated the Ear and Hearing Disorders Survey Protocol in 1999. While hearing screening is routinely carried out among school-age children in developed countries, in some developing countries, there is not a consummate procedure to prevent or treat ear diseases promptly and effectively. In China, a developing country with the highest population in the world, hearing disabilities have been one of the most common types of disabilities. The second national disability survey in 2006 reported a hearing disabled population of 27.8 million in China. In two investigations conducted
C Biomed Central

() 2011 Chen et al; licensee BioMed Central Ltd. This is an Open Access article distributed under the terms of the Creative Commons Attribution License (http://creativecommons.org/licenses/by/2.0), which permits unrestricted use, distribution, and reproduction in any medium, provided the original work is properly cited. 
in eastern China, $10 \%$ of 442 urban students and $20 \%$ of 282 rural students, ranging from 6 to 19 years of age, were diagnosed as $\mathrm{HI}[7,8]$.

A recent study showed that portable audio deviceinduced hearing loss was evolving into a significant social and public health problem [9]. HI contributes not only to academic difficulty but also to sleep impairment, especially insomnia [10]. Therefore, it is important to explore the association of SHL with study stress, insomnia and the use of portable audio devices. In this study, we determined the prevalence of ear disease among secondary school students in Xi'an, a provincial capital city in western China. Furthermore, related social behaviors and their associations with SHL were investigated and are discussed below.

\section{Methods \\ Participants}

This cross sectional study, conducted between April 2009 and July 2009, investigated the distribution of ear diseases among secondary school students in Xi'an. Particular attention was paid to the prevalence and characteristics associated with SHL. Students from 29 secondary schools in Xi'an were selected using a stratified sampling method. Ten schools were randomly selected from the 29 schools, and three grades of each school were selected according to a random table. The total number of participants was 1567 (541, 557 and 469 in grades eight, ten, and eleven, respectively).

This study was approved by the Institutional Review Board of the Ethics Committee of Xijing Hospital, the Fourth Military Medical University of the People's Liberation Army of China. Written informed consents were obtained from all families and participants.

\section{Otoscopy}

Each ear was examined by pneumatic otoscopy. All abnormal findings were confirmed by a trained otolaryngologist.

\section{Audiometry}

The pure tone test was performed in a sound-proof audiometric room in Xijing Hospital (OB922 pure tone audiometer, Madsen, Denmark, bio-calibrated daily). Air conduction was performed for 250, 500, 1000, 2000, 4000 , and $8000 \mathrm{~Hz}$. Bone conduction was performed for 250, 500, 1000, 2000, and $4000 \mathrm{~Hz}$. All tests followed the Hughson-Westlake ascending method. Narrow band noise masking was applied to the opposite ear when the difference in threshold was no less than either $40 \mathrm{~dB}$ between the air conduction of the test and non-test ear or $15 \mathrm{~dB}$ between the air and bone conduction of the test ear.

\section{Tympanometry}

Tympanometry was performed with the acoustic impedance instrument ZODIAC901 (Madsen, Denmark). Tympanic compliance, middle ear pressure, and tympanogram were recorded.

\section{Questionnaire}

A survey detailing each student's demographics, medical history, and noise exposure was administered. With respect to noise exposure, the average use time and volume of portable audio devices was recorded. Two validated questionnaires, the Chinese Mental Health Inventory for Middle School Students (CMHI) [11] and the Athens insomnia scale (AIS) [12], were conducted to reflect academic stress and insomnia.

Sleep (reflected by AIS) was defined as normal (0-3 points), probable sleeping disorder (4-5 points), or insomnia ( $\geq 6$ points).

According to the exposure level, portable audio device use was categorized as control (without a portable audio device), low exposure (less than 0.5 hour per day), medium exposure (0.5-1.5 hours per day), and high exposure (more than 1.5 hours per day). "Loud sound" ( $\geq 85 \mathrm{~dB}$ ) was defined as frequently using more than two-thirds of the maximum volume.

\section{Diagnostic criteria}

According to the $\mathrm{WHO} /$ International Organization for Standardization (ISO) definition, participants were diagnosed as HI when the average threshold (500, 1000, 2000 , and $4000 \mathrm{~Hz}$ ) was more than 25 decibels of hearing loss (dB HL) in either ear. The severity of HI (based on the pure tone average in the better ear) was categorized as mild (26-40 dB HL), moderate (41-60 dB HL), severe (61-80 dB HL), or profound (> $80 \mathrm{~dB} \mathrm{HL}$ ). HI was further classified as conductive, sensorineural, or mixed.

Before investigating the association between SHL and portable audio device use, we excluded the subjects with middle ear disease, congenital deafness, drug-induced deafness, history of head injury, familial deafness, or other ear organic disease.

\section{Statistical analysis}

The data were entered into EpiData 3.0 software and verified by two of the authors. The chi-square test was performed to show the association of SHL with related behaviors. All variables (gender, age, portable audio device use, academic stress, sleep disorder, history of ototoxic drug administration and family history of deafness) were included in a logistic regression model to identify correlation factors for HI. Odds ratios and 95\% confidence intervals were calculated (SPSS version 15.0; 
SPSS Inc., Chicago, Ill.). A P-value of less than 0.05 was considered statistically significant.

\section{Results}

\section{Percentage of identified ear diseases}

A total of 1567 students (793 boys, 774 girls) were enrolled between April 2009 and July 2009. The ages ranged from 12 to 19 with a mean of 15.7 years. Following the WHO definitions, 23 of 1567 participants had mild HI, 4 had moderate HI, 2 had severe HI, and 1 had profound HI (Table 1). Among the students with impaired hearing, 4 had conductive hearing impairment loss (23.33\%), 3 had mixed hearing loss (10\%), and 23 had SHL (66.67\%). Bilateral hearing loss was detected in 19 of the 30 students with HI.

The percentage of students with ear disease was 3.32\% in total. External ear disease, middle ear disease and SHL accounted for $1.21 \%, 0.64 \%$, and $1.47 \%$, respectively (Table 2). As shown in Table 3 the percentage of ear disease among boys (5.30\%) was significantly higher than that among girls $(1.29 \%, P<0.001)$. The risk for the former was 4.273-fold higher than the latter $(95 \%$ confidence interval 2.128-8.578). There was no significant difference in the percentage of ear disease among different ages.

In a multiple logistic regression model (Table 4), gender, use of portable audio devices, sleep disorders, history of ototoxic drug administration and family history of deafness were associated with HI, while age was not. In addition, use of portable audio devices was an

Table $1 \mathrm{HI}$ of different level

\begin{tabular}{ccc}
\hline Ear diseases & Number & $\begin{array}{c}\text { Percentage } \\
\text { (\%) }\end{array}$ \\
\hline Mild (26 - $4 \mathrm{~dB}$ ) & 23 & 1.47 \\
External auditory canal stenosis & 1 & \\
Active stage of chronic suppurative otitis & 3 & \\
media & & \\
Secretory otitis media & 1 & \\
Tympanic membrane perforation (dry ear) & 1 & \\
Tympanosclerosis & 1 & \\
Hereditary & 3 & \\
Noise-induced & 12 & \\
Unknown & 1 & \\
Moderate (41 - $60 \mathrm{~dB})$ & 4 & \\
Tympanosclerosis & 1 & \\
Hereditary & 1 & \\
Noise-induced & 2 & \\
Severe (61 - $80 \mathrm{~dB})$ & 2 & \\
Drug-induced & 1 & \\
Unknown & 1 & \\
Profound (> $80 \mathrm{~dB})$ & 1 & \\
Unknown & 1 & \\
\hline
\end{tabular}

Table 2 Percentage of identified ear diseases

\begin{tabular}{ccc}
\hline Ear diseases & Number & $\begin{array}{c}\text { Percentage } \\
\text { (\%) }\end{array}$ \\
\hline External ear diseases & 19 & 1.21 \\
Accessory auricle & 7 & 0.45 \\
Congential preauricular fistula & 8 & 0.51 \\
External auditory canal stenosis & 2 & 0.13 \\
Otisis externa & 1 & 0.06 \\
First branchial cleft fistula & 1 & 0.06 \\
Middle ear disease & 10 & 0.64 \\
Active stage of chronic supprative otisis & 3 & 0.19 \\
media & 2 & \\
Secretory otitis media & 3 & 0.13 \\
Tympanic membrane perforation(dry ear) & 2 & 0.19 \\
Tympanosclerosis & 23 & 1.47 \\
Sensorineural hearing loss & 4 & 0.26 \\
Hereditary & 1 & 0.06 \\
Drug-induced & 14 & 0.89 \\
Noise-induced & 4 & 0.26 \\
Unknown &
\end{tabular}

independent risk factor of HI. Female gender was a protective factor for HI.

\section{SHL-related social behaviors}

SHL was not associated with academic stress scores $\left(\chi^{2}\right.$ $=4.065, \mathrm{P}=0.255)$ (Table 5). However, it was significantly increased among those students with a sleeping disorder and insomnia $\left(\chi^{2}=9.761, \mathrm{P}=0.008\right)$ (Table 6). By logistic regression analysis, we found a greater risk of SHL when the insomnia score was more than $6(\mathrm{P}=$ 0.002, $\alpha=0.015$ ).

According to our survey, 1335 (85.19\%) students used portable audio devices. Among these subjects, 1098 $(82.20 \%)$ students preferred loud sound ( $\geq 85 \mathrm{~dB})$. The average use time was $1.41 \pm 1.11$ hours per day. SHL was associated with the extent of portable audio device use $\left(\chi^{2}=8.15, P=0.043\right)$ and they were positively correlated (Figure 1).

\section{Intervention needed}

According to the degree of $\mathrm{HI}$, different interventions were advised: mild HI warranted a consultation and consideration of a hearing aid; for moderate HI a hearing aid was recommended; severe HI warranted a hearing aid, lip reading, and sign language instruction; and profound HI warranted a hearing aid, rehabilitation, lip reading, and sign language instruction. In total, 80 participants $(5.11 \%)$ needed medical interventions. Hearing aids were needed by 23 students (1.47\%). Non-emergency ear surgeries were required for 14 students $(0.89 \%)$ (10 cases of middle ear disease, 2 cases of congenital preauricular fistula with infection history and 2 
Table 3 Percentage of ear disease stratified by gender and age

\begin{tabular}{cccccc}
\hline \multicolumn{2}{c}{ Gender and Age } & Ear disease & Without ear disease & Total & Percentage of ear disease (\%) \\
\hline Gender $^{1}$ & Boy & 42 & 751 & 793 & $5.30 \%$ \\
& Girl & 10 & 764 & 774 & $1.29 \%$ \\
Age $^{2}$ & $12-14$ & 13 & 408 & 421 & $3.09 \%$ \\
& $15-17$ & 36 & 993 & 1029 & $3.50 \%$ \\
& $18-19$ & 3 & 114 & 117 & $2.56 \%$ \\
\hline
\end{tabular}

$X^{2}{ }_{1}=19.576, P=0.000 ; X^{2}{ }_{2}=0.381, P=0.826$

cases of external auditory canal stenosis). Additionally, 29 boys (3.66\%) and 13 girls (1.68\%) had cerumen impaction, and they all needed cerumen removal. One student with otitis externa needed medical treatment. One student with profound SHL needed speech rehabilitation.

\section{Discussion}

This is the first study investigating the prevalence of ear diseases among Chinese adolescents using a stratified sampling method. Although it is only a local and partial survey, the current situation of ear diseases among urban secondary school students in Northwest China can be extrapolated. This survey provides scientific evidence for China and other developing countries to develop adolescent HI prevention and control strategies.

The observation that $3.32 \%$ percent of students have some form of ear disease suggests an urgent need for otologic health care in adolescents. Many of the cases affected the external ear, including the accessory auricle, the congenital preauricular fistula, the external auditory canal stenosis, the otitis externa and the first branchial cleft fistula. Although external ear diseases usually do not cause direct hearing and balancing damage, they may place a child at risk for developing damage. Middle ear disease was represented mainly by chronic purulent otitis media and secretory otitis media. The small proportion $(19.23 \%, 10 / 52)$ of middle ear diseases is probably ascribed to the extensive application of antibiotics and the decreased incidence of secretory otitis media

Table 4 Multiple logistic regression analysis of $\mathrm{HI}$

\begin{tabular}{|c|c|c|c|}
\hline Variable & $\begin{array}{c}O R \\
\text { value }\end{array}$ & $95 \% \mathrm{Cl}$ & $\begin{array}{c}P \\
\text { value }\end{array}$ \\
\hline Gender & 0.342 & $0.250-0.638$ & 0.004 \\
\hline Age & 1.350 & $0.555-3.284$ & 0.532 \\
\hline Portable audio device use & 8.676 & $\begin{array}{l}5.176- \\
12.462\end{array}$ & 0.026 \\
\hline Academic stress & 5.006 & $\begin{array}{l}0.984- \\
11.280\end{array}$ & 0.095 \\
\hline Sleep disorder/insomnia & 1.925 & $1.040-4.377$ & 0.035 \\
\hline $\begin{array}{l}\text { History of ototoxic drug } \\
\text { administration }\end{array}$ & 2.316 & $1.071-5.258$ & 0.046 \\
\hline Family history of deafness & 3.518 & $1.124-4.334$ & 0.009 \\
\hline
\end{tabular}

with physical development. However, the excessive treatment of secretory otitis media may lead to tympanic sclerosis during childhood [13], and, in fact, we observed two cases of tympanic sclerosis in our study. Therefore, the long-term effects of treatment should be carefully considered when making a surgical decision. The low proportion of chronic purulent inflammation we observed was consistent with the survey by Zakzouk et al. [14], which showed a decrease in urban incidence of chronic suppurative otitis media (CSOM) over the past two decades. Nevertheless, we need to remain vigilant because of the severe extracranial and intracranial complications due to chronic purulent inflammation. The majority of people were not aware of these ear diseases. Therefore, it is important to put some effort into otologic health education among school students.

In this survey, the most common ear disease $(44.23 \%$, 23/52) was SHL caused by noise, drugs, heredity, CSOM, otosclerosis and unidentified factors. SHL can cause life-long and sometimes life-threatening difficulties and it can profoundly affect the ability to communicate as well as hinder education and employment. Therefore, education on ear health deserves more attention in schools.

Congenital ear disease accounts for $42.31 \%$ (22/52) of the ear diseases we observed. It causes concern not only because of the possible damage to hearing or balance, but also due to its heritability and association with hereditary diseases in other organs.

According to our survey, the percentage of ear disease among boys was significantly higher than that among girls. While this is consistent with a study from the Jiangsu Province of China [7], it is contrary to that of Shargorodsky, et al. in 2010 [15]. The discrepancy may

\section{Table 5 Percentage of SHL stratified by academic stress} level

\begin{tabular}{ccccc}
\hline Academic stress & SHL & Without SHL & Total & Percentage of SHL(\%) \\
\hline Normal & 14 & 922 & 936 & 1.50 \\
Mild & 6 & 514 & 520 & 1.15 \\
Moderate & 2 & 96 & 98 & 2.04 \\
Severe & 1 & 12 & 13 & 7.69 \\
\cline { 2 - 5 } Total & 23 & 1544 & 1567 & 1.47 \\
\hline
\end{tabular}

$X^{2}=4.065, P=0.255$ 
Table 6 Percentage of SHL stratified by sleep problems

\begin{tabular}{ccccc}
\hline AIS Score & SHL & Without SHL & Total & Percentage of SHL(\%) \\
\hline Normal & 19 & 1282 & 1301 & 1.46 \\
Sleeping disorder & 3 & 257 & 260 & 1.15 \\
Insomnia & 1 & 5 & 6 & 16.67 \\
\cline { 2 - 5 } Total & 23 & 1544 & 1567 & 1.47 \\
\hline
\end{tabular}

$X^{2}=9.761, P=0.008$

result from differences in the genetic backgrounds of the study populations or the increased difference in use of portable audio and gaming devices by boys compared to girls in China. HI didn't correlate with age in our study, which may be a result of the minimal age difference of the subjects.

The percentage of students with SHL did not differ among the students with elevated levels of academic stress. However, because $40.27 \%$ of the students reported experiencing at least mildly elevated levels of academic stress, schools and communities may want to address this issue. The percentage of SHL increased significantly among those students with insomnia. Because there is not enough evidence to decide the exact causality, further studies are necessary to determine the relationship between insomnia and SHL.

Among all causes of SHL, noise was the most common $(60.87 \%, 12 / 23)$. With the economic development of China, industrial, transportation-related and entertainment-related noise pollution has become increasingly prominent. While a temporary threshold shift in noise induced hearing loss is reversible, it is difficult to stop the progression of the threshold shift from temporary to permanent. It was recently reported that $3.4 \%$ of female and $10.3 \%$ of male youths in the US used hearing-protection in 2005-2006 [16]. However, the students in our study lacked awareness of hearing protection principles. Therefore, an important method for reducing $\mathrm{HI}$ is to provide hearing health care education and

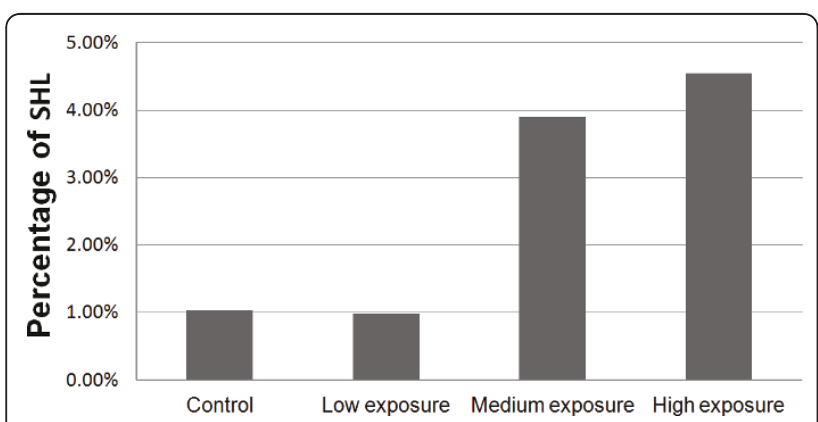

Figure 1 Percentage of SHL among students who use portable audio devices. The percentage of students with SHL in control, low exposure, medium exposure and high exposure strata were 1.04\%, $0.99 \%, 3.91 \%$ and $4.55 \%$, respectively. guide adolescents to use portable audio and gaming devices appropriately.

This study only investigated ear diseases among urban and suburban students, not rural students. Studies covering a more extensive and varied area are needed. In addition, otologic eikonic data, important for the etiological diagnosis of SHL, were not collected.

This study suggests that the education and popularization of hearing health knowledge among adolescents should be prioritized. Otologists should reinforce the prevention and rehabilitation of $\mathrm{HI}$ among young people while technical services and the appropriate help should be provided to people with hearing and speech disabilities. Cerumen impaction accounted for a significant proportion of ear problems. Although cerumen removal should be carried out in a hospital, non-medical cerumen removal was very common. This practice, and the excessive use of portable audio devices, are two examples of bad habits those should be corrected to avoid the potential risk of $\mathrm{HI}$.

\section{Conclusions}

More attention should be paid to the high prevalence of ear disease among secondary school students. Insomnia and the excessive use of portable audio devices may be associated with adolescent SHL. It is important to establish and comply with an evidence-based preventive strategy.

\section{Acknowledgements}

We would like to thank 1567 volunteer students, their parents and their teachers for their active participation, and government departments who provided support. In addition, we would like to thank Yu-Xiang Bai (Statistics Department, Fourth Military Medical University) who provided constructive statistical advice.

This study was supported by grants from the National Natural Science Foundation of China (30600703, 30973299).

\section{Authors' contributions}

JQ initiated and designed the survey. YC was responsible for the organization of the survey and the division of labor. $X L$ was in charge of data collection and manuscript drafting. ZX, ZL, PZ, YH and FW performed the otologic examinations and administered the questionnaires. All authors read and approved the final manuscript. JQ is guarantor of the paper.

\section{Competing interests}

The authors declare that they have no competing interests.

Received: 23 February 2011 Accepted: 8 June 2011

Published: 8 June 2011

\section{References}

1. Bess FH, Tharpe AM: Unilateral hearing impairment in children. Pediatrics 1984, 74:206-216.

2. Bess FH, Dodd-Murphy J, Parker RA: Children with minimal sensorineural hearing loss: prevalence, educational performance, and functional status. Ear Hear 1998, 19:339-354.

3. Lieu JE: Speech-language and educational consequences of unilateral hearing loss in children. Arch Otolaryngol Head Neck Surg 2004, 130:524-530. 
4. Khairi Md Daud M, Noor RM, Rahman NA, Sidek DS, Mohamad A: The effect of mild hearing loss on academic performance in primary school children. Int I Pediatr Otorhinolaryngol 2010, 74:67-70.

5. Primary ear and hearing care training resource advanced level. [http:// www.who.int/entity/pbd/deafness/activities/hearing_care/advanced.pdf].

6. White KR, Forsman I, Eichwald J, Munoz K: The evolution of early hearing detection and intervention programs in the United States. Semin Perinatol 2010, 34:170-179.

7. Morioka I, Luo WZ, Miyashita K, Takeda S, Wang YX, Li SC: Hearing impairment among young Chinese in a rural area. Public Health 1996, 110:293-297.

8. Kaewboonchoo O, Morioka I, Miyashita K, Takeda S, Wang YX, Li SC: Hearing impairment among young Chinese in an urban area. Public Health 1998, 112:143-146.

9. Vogel I, Brug J, Van der Ploeg CP, Raat H: Adolescents risky MP3-player listening and its psychosocial correlates. Health Educ Res 2011, 26:254-264.

10. Test T, Canfi A, Eyal A, Shoam-Vardi I, Sheiner EK: The influence of hearing impairment on sleep quality among workers exposed to harmful noise. Sleep 2011, 34:25-30.

11. Que M, Tao FB, He CY, Zhang LH, Zhu P: Study on the relationship between mental health and overweight, obesity, different sex among junior middle school students. Zhonghua Liu Xing Bing Xue Za Zhi 2007, 28:460-463.

12. Lazaratou H, Dikeos DG, Anagnostopoulos DC, Sbokou O, Soldatos CR: Sleep problems in adolescence. A study of senior high school students in Greece. Eur Child Adolesc Psychiatry 2005, 14:237-243.

13. Yaman H, Guclu E, Yilmaz S, Ozturk O: Myringosclerosis after tympanostomy tube insertion: relation with tube retention time and gender. Auris Nasus Larynx 2010, 37:676-679.

14. Zakzouk SM, Hajjaj MF: Epidemiology of chronic suppurative otitis media among Saudi children-a comparative study of two decades. Int J Pediatr Otorhinolaryngol 2002, 62:215-218.

15. Shargorodsky J, Curhan SG, Curhan GC, Eavey R: Change in prevalence of hearing loss in US adolescents. JAMA 2010, 304:772-778.

16. Henderson E, Testa MA, Hartnick C: Prevalence of noise-induced hearingthreshold shifts and hearing loss among US youths. Pediatrics 2011, 127: e39-46.

\section{Pre-publication history}

The pre-publication history for this paper can be accessed here: http://www.biomedcentral.com/1471-2458/11/445/prepub

doi:10.1186/1471-2458-11-445

Cite this article as: Chen et al: Ear diseases among secondary school students in Xi'an, China: The role of portable audio device use, insomnia and academic stress. BMC Public Health 2011 11:445.

\section{Submit your next manuscript to BioMed Central and take full advantage of:}

- Convenient online submission

- Thorough peer review

- No space constraints or color figure charges

- Immediate publication on acceptance

- Inclusion in PubMed, CAS, Scopus and Google Scholar

- Research which is freely available for redistribution

Submit your manuscript at www.biomedcentral.com/submit
Biomed Central 